León Carrascosa, V. y Fernández-Díaz, M.J. (2019). Diseño y validación de una escala para evaluar el funcionamiento de las tutorías en Educación Secundaria. Revista de Investigación Educativa, 37(2), 525-541.

DOI: http://dx.doi.org/10.6018/rie.37.2.345251

\title{
Diseño y validación de una escala para evaluar el funcionamiento de las tutorías en Educación Secundaria
}

\section{Design and validation of a scale to evaluate the functioning of tutoring in Secondary Education}

\author{
Víctor León Carrascosa y María José Fernández-Díaz \\ *Departamento de Investigación y Psicología en Educación. Facultad de Educación. Universidad Complutense de \\ Madrid
}

\begin{abstract}
Resumen
El desarrollo de la función tutorial es clave para la formación integral de los estudiantes. En los últimos años las investigaciones sobre este tema se centran en la educación superior relegando a segundo plano su desarrollo en la educación básica. En este sentido, el presente trabajo tiene como objetivo diseñar y validar un instrumento de medida sobre la valoración de las tutorías en Educación Secundaria Obligatoria y Posobligatoria, fundamentado teóricamente, que reúna las características psicométricas exigidas: fiabilidad, homogeneidad de los items y validez. La muestra del estudio está compuesta por 4254 estudiantes de Educación Secundaria Obligatoria y Postobligatoria correspondientes a los diferentes tipos de centros educativos de la Comunidad Autónoma de Madrid (CAM). A raíz de la literatura consultada y la experiencia profesional se consideraron cuatro dimensiones sobre la práctica tutorial donde se analizó la fiabilidad (Alfa de Cronbach total del instrumento: 0.929, y por dimensiones, Funciones con el alumnado: 0.868; Funciones con las familias: 0.649; Desarrollo: 0.797; Evaluación: 0.876), los valores del coeficiente de discriminación de los ítems (datos superiores a 0.2, según Hair et al., 2014) y el Análisis Factorial Confirmatorio ( $\chi 2 / g l=0.0254$; CFI= 0.940; RMSEA: 0.044; $P R A T I O=0.894$ ) obteniendo resultados satisfactorios. Por todo ello, el instrumento propuesto contribuye al estudio de las tutorías, tanto desde un punto de vista teórico como por sus características psicométricas relativas a su fiabilidad y validez. De igual modo, el instrumento
\end{abstract}

Correspondencia: Víctor León Carrascosa, victorleoncarrascosa@ucm.es, Departamento de Investigación y Psicología en Educación. Facultad de Educación. Universidad Complutense de Madrid. 
se constituye como una herramienta de utilidad en el desarrollo de las tutorías, atendiendo las demandas y necesidades de sus estudiantes.

Palabras clave: tutoría; educación básica; evaluación; instrumento de medida.

\begin{abstract}
The development of the tutorial function development is the key for an integral formation of the students. In recent years, research on this topic has been focused on higher education, relegating to a second place its development in basic education. In this sense, the objective of this work is to design and validate a measuring instrument for the assessment of tutorials in Obligatory and Post-obligatory Secondary Education, supported theoretically, that finds the required psychometric characteristics: reliability, homogeneity of the items and validity. The study sample is made up of 4254 students of Compulsory and Post-compulsory Secondary Education corresponding to different types of educational centers in Autonomous Community of Madrid (CAM). As a result of the literature consulted and the professional experience, four dimensions were considered on the tutorial practice where the reliability was analyzed (Alfa of Cronbach total of the instrument: 0.929, and by dimensions, Functions with the students: 0.868; Functions with the families: 0.649; Development: 0.797; Evaluation: 0.876), the values of the discrimination coefficient of the items (data superior to 0.2, according to Hair et al. 2014) and the Confirmatory Factorial Analysis ( $\chi 2 / g l=0.0254 ; C F I=0.940 ;$ RMSEA: 0.044; $P R A T I O=0.894$ ) obtaining satisfactory results. For all these reasons, the proposed instrument contributes to the study of tutorials, both from a theoretical point of view and because of their psychometric characteristics relative to their reliability and validity. Similarly, the instrument constitutes a useful tool in the development of tutorials, meeting the students' demands and needs.
\end{abstract}

Keywords: tutorial; basic education; evaluation; measuring instrument.

\title{
Introducción
}

A lo largo de la historia los centros educativos han venido siendo lugares destinados a la formación de las personas, ya no solo en el plano intelectual, sino también en el desarrollo formativo de las mismas. Sus estructuras organizativas difieren en muchos casos del contexto social en el que se encuentran inmersos, primando la formación de los estudiantes, tanto en el ámbito intelectual y cognitivo, como en otros aspectos relacionados con la formación humana y social. Igualmente, los centros están ligados al contexto familiar del alumnado, siendo un apoyo de los valores transmitidos en él. En este sentido, es importante reconocer la relevancia de la orientación educativa, y concretamente la tutoría, para guiar a los estudiantes y a sus familias como elemento fundamental en el acompañamiento continuo y personalizado de cada estudiante, garantizando su desarrollo integral en todos los ámbitos (académico, social, personal y profesional) (González-Benito \& Vélaz de Medrano, 2014).

La tutoría se convierte en una herramienta de la que disponen los centros educativos y los propios docentes, para mejorar sustancialmente la calidad de los procesos educativos. Sin embargo, se concede mayor importancia a la tarea académica que a la formativa de la persona (Rodríguez Fernández \& Romero, 2015). 
Asimismo, la mayoría de las investigaciones sobre esta temática se centran en la educación universitaria, relegando a segundo plano las investigaciones en la educación básica como eje medular de la formación integral de los estudiantes, donde su aprendizaje requiere de procesos sistemáticos para un mejor desarrollo humano. De la Cruz y Abreu-Hernández (2017) comprueban la carencia de instrumentos para evaluar la tutoría en relación con las funciones y actividades del proceso formativo. Sin embargo, aunque centrados la mayoría en la educación superior, si hemos podido comprobar algunos estudios que utilizan instrumentos para evaluar las necesidades formativas de los orientadores (Anaya, Pérez-González, \& Suárez, 2011), los programas de acción tutorial y el análisis sobre si el departamento al que pertenece el profesor influye en dicha evaluación (Sáiz-Manzanares, Bol-Arreba, \& Payo-Hernanz, 2014), la función orientadora del profesorado (Arza, De Salvador, \& Mascarenhas, 2014), la satisfacción con la tutoría (Pérez Cusó, Martínez Clares, \& Martínez Juárez, 2015), la satisfacción de las familias sobre las tutorías (Castellano \& Pantoja, 2015; López \& Pantoja, 2016), las actitudes hacia la tutoría de los estudiantes (Caldera, Carranza, Jiménez, \& Pérez, 2015; Solaguren-Beascoa \& Moreno, 2016), la competitividad de los programas de tutorías (Rosiles, Macías, Pérez, \& León, 2013) y la evaluación de posibles recursos para poner en marcha la acción tutorial con la finalidad de programar y planificar su desarrollo (Rodríguez-Fernández \& Romero, 2015).

Para cumplir con el propósito de nuestro estudio es necesario partir de la fundamentación teórica. Por ello, de acuerdo a la literatura consultada, el estudio de diferentes herramientas sobre las tutorías y desde nuestra experiencia profesional, hemos tratado de generar una estructura de cuatro dimensiones que abarque los siguientes aspectos sobre la práctica tutorial en las aulas: Funciones del Tutor con el Alumnado, Funciones del Tutor con las Familias, Desarrollo de las Tutorías y Evaluación de las Tutorías, que se justifican a continuación.

En cuanto a las Funciones del Tutor con el Alumnado son diversas las investigaciones que recogen las cualidades necesarias que debe poseer un tutor (García Nieto, 2011; Jiménez \& Navaridas, 2012; Youde, 2016). El tutor representa una figura influyente en las relaciones y las formas de enseñar, siendo un modelo guía de conducta y actitud, facilitador del diálogo para dar respuestas a cuestiones de muy diverso tipo, bien sean escolares, personales o profesionales (Alegre, Guzmán \& Arvelo, 2017). Pérez-Cusó et al. (2015) destacan la accesibilidad, la creación de un clima favorable, la disposición y las habilidades interpersonales del profesorado, y Hudson, Usak \& Savran-Gencer (2009) añaden el manejo del grupo en el aula. Igualmente, Gil-Albarova, Martínez, Tunnicliffe y Moneo (2013) valoran los aspectos emocionales (el trato humano, el interés, la atención personalizada) siendo el tutor ideal aquel que apoya en el proceso de enseñanza-aprendizaje y que impulsa simultáneamente el desarrollo personal y profesional. Sáiz-Manzanares et al. (2014) se centran en las mejoras de las interacciones de los alumnos con los docentes y en las funciones tutoriales del profesor, valorando el interés para desempeñar el papel del profesor tutor. En esta misma línea, la responsabilidad de ejercer la función del tutor debe ir ligada a los conocimientos sobre su profesión y una madurez personal y emocional adecuada, ya que, dentro de sus cometidos, está el orientar a otras personas en el proceso de maduración (González-Benito \& Vélaz de Medrano, 2014; Martínez-Clares, Martínez Juárez, Pérez Cusó, González Morga, \& González Lorente, 2016). 
Las Funciones del Tutor con las Familias resultan especialmente complejas pero importantes para el desarrollo integral de los estudiantes. Autores como Álvarez Justel (2017), González-Benito y Vélaz de Medrano (2014) y Urosa y Lázaro (2017) valoran la información y asesoramiento que reciben las familias a través de la organización de reuniones informativas, el análisis del progreso educativo de sus hijos, el fomento de su participación en el centro educativo y la implicación en actividades de apoyo al aprendizaje y orientación de los hijos, además de contribuir en el establecimiento de relaciones fluidas entre ambos contextos socioeducativos. López y Pantoja (2016) señalan aspectos como el grado de satisfacción familiar con la acción tutorial, la implicación de las familias en la tutoría, el conocimiento de las funciones y tareas de la acción tutorial y el uso de las TIC. Del mismo modo, Castellano y Pantoja (2015) indican que las familias aceptan el empleo de los recursos tecnológicos y aquellas actividades que contribuyen a desarrollar las habilidades sociales, los hábitos de trabajo, el ocio y las actitudes hacia los demás en la formación de sus hijos.

En la dimensión Desarrollo de las tutorías en el centro educativo y el aula se pone especial énfasis en los programas centrados en la promoción de la igualdad y el respeto a la diversidad socio-afectiva y sexual, la educación mixta, la igualdad de género, las relaciones en pareja, entre otros (Díaz de Greñu \& Parejo, 2013; Sánchez-Porro \& González, 2017). Son aspectos claves en que la escuela debe formar como parte del progreso personal del estudiante. Asimismo, se ha de considerar el desarrollo de programas que favorezcan la educación en valores (Lozano, 2012), la comprensión y expresión de las emociones (educación emocional), el desarrollo de habilidades para la vida, como las sociales, la prevención de consumo de drogas, la educación para la salud, etc. (Álvarez González \& Bisquerra, 2012). Por otro lado, se debe ayudar a reflexionar, a escuchar atentamente y a organizarse (Hudson et al., 2009) a través del trabajo de actividades que promuevan el desarrollo profesional y académico (Pérez-Cusó et al., 2015). Santana, García y Cruz (2010) indican que se tiene que propiciar en los estudiantes la capacidad de toma de conciencia, conocer e identificar sus propias metas y la búsqueda de información laboral y académica, permitiendo conocerse a sí mismo, decidiendo su itinerario profesional, académico y de vida (Sánchez García, 2013).

La Evaluación de las tutorías se centra en la información necesaria que se obtiene a lo largo del proceso para encauzarlo de una manera u otra y la necesidad de mejorar, cambiar o continuar con la acción planificada (Monge, 2010). Otros autores hacen mención a la evaluación de las relaciones entre el profesorado y los estudiantes atendiendo a la satisfacción de los estudiantes en las tutorías, la valoración de los temas y cuestiones desarrolladas en el grupo clase, la implicación del alumnado en las actividades propuestas y la necesidad de contar con un clima de aula adecuado, alcanzando un grado de estima, confianza y aceptación mutua entre tutores y alumnos (Aguaded, 2014). Igualmente, Rubio y Martínez (2012) destacan el valor que se atribuye a los procesos de diagnóstico y evaluación en los que cuentan o no con la participación de los estudiantes, siendo motivo de éxito o fracaso para los diferentes programas de acción tutorial. Por último, Torrecilla, Rodríguez, Herrera y Martín (2013) apuntan cómo el éxito percibido por los estudiantes se relaciona con aspectos de tipo personal (conocimiento de compañeros, satisfacción con las sesiones de tutoría, valoración, funcionalidad...). 
En este contexto y debido a la carencia de estudios sobre la acción tutorial en niveles educativos que no sean de educación universitaria, el presente trabajo tiene como principal objetivo diseñar y validar un instrumento de medida sobre la valoración de las tutorías por parte de los estudiantes de los niveles educativos a los que nos referimos, fundamentado teóricamente, que reúna las características psicométricas exigidas: fiabilidad, homogeneidad de los ítems y validez. Partiendo de la fundamentación teórica nuestro instrumento ha considerado 4 dimensiones: Funciones del Tutor con el Alumnado, Funciones del Tutor con las Familias, Desarrollo de las Tutorías y Evaluación de las Tutorías.

\section{Método}

\section{Muestra}

La población de nuestro estudio está conformada por los estudiantes de Educación Secundaria Obligatoria y Postobligatoria correspondientes a los diferentes centros educativos de las distintas zonas de la Comunidad Autónoma de Madrid (CAM). El tipo de muestra seleccionado, dentro del muestreo No Probabilístico, fue Incidental, tratando de garantizar la representatividad de los estudiantes. De este modo, el 55.5\% pertenecen a la zona centro, el $22.22 \%$ zona sur, el $11.11 \%$ zona oeste, el $5.56 \%$ zona este y el $5.56 \%$ a la zona norte. Esta distribución se ajusta adecuadamente a la población total de las zonas de la CAM: $51.36 \%$ zona centro, $19.72 \%$ zona sur, el $12.58 \%$ zona oeste, el $9.48 \%$ zona este y el $6.86 \%$ a la zona norte (Estadística de la enseñanza de la CAM, 2016). En cuanto al tipo de centro, el $32.1 \%$ pertenecen a centros públicos, el $44.9 \%$ centros concertados y el $23 \%$ a centros privados.

Por otro lado, la participación de los sujetos fue voluntaria, obteniendo una muestra de 4254 estudiantes (2127 mujeres y 2127 hombres) pertenecientes a 18 centros. El $54.2 \%$ de los estudiantes tienen 14 años o menos, el 32.8\% entre 15 - 16 años y el 13\% tienen 17 años o más. Respecto a la distribución por cursos, el $21.8 \%$ están en $1^{\circ}$ ESO, el $22.1 \%$ en $2^{\circ}$ ESO, el $19.4 \%$ en $3^{\circ}$ ESO, el $19.8 \%$ en $4^{\circ}$ ESO y el $16.9 \%$ en Bachillerato. Como podemos observar, la muestra cumple los requisitos establecidos por Hair, Black, Babin y Anderson (2014) para la realización de los análisis de validez de constructo, contando con un mínimo número de observaciones cinco veces mayor que el número de variables a estudiar. Por consiguiente, nuestra muestra está formada por 4254 observaciones y el instrumento de medida está configurado por 37 ítems, obteniendo un ratio de observaciones /variables de 66.32, situado por encima de lo recomendado.

\section{Instrumento}

Resaltamos la importancia de este subapartado dado que el objetivo de nuestro estudio es aportar un instrumento técnicamente elaborado para valorar el funcionamiento de las tutorías por parte de los estudiantes. Dicho instrumento está formado por ítems que responden a las cuatro dimensiones mencionadas: Funciones del tutor con el alumnado, Funciones del tutor con las familias, Desarrollo de la tutoría y Evaluación de la misma. De esta manera, el cuestionario quedó configurado por 37 ítems (ver tabla 1) a 
los que los estudiantes debían responder atendiendo a una escala de 1 a 5 tipo Likert, donde 1 indica Nada/Casi Nada y 5 Totalmente/Mucho.

\section{Elaboración del cuestionario}

La elaboración de la escala para evaluar las tutorías comprende cuatro grandes dimensiones que refleja la fundamentación teórica y la coherencia con los elementos contemplados en el instrumento. A continuación, se detalla la descripción de las cuatro dimensiones.

La dimensión Funciones con el alumnado se centra en aspectos como la formación del tutor, la atención del alumnado, la mediación en el aula y las relaciones entre el profesorado y los estudiantes y con los demás. La dimensión Funciones con la familia visiona el modo de participación y colaboración entre ambos contextos y la atención recibida por las familias desde las tutorías sobre sus hijos. La dimensión Desarrollo recoge diferentes tipos de actividades, ya sean tradicionales e innovadoras, tales como la tecnología y la colaboración externa, conociendo el trabajo de la orientación desde la comunicación, las diferencias individuales y la toma de decisiones. Por último, la dimensión Evaluación comprende las valoraciones personales en torno a las tutorías, sus dinámicas distintivas en su funcionamiento y la mejora y transferencia de las tutorías.

Para obtener evidencias de validez de contenido del instrumento en relación con la fundamentación teórica se utilizó el procedimiento de juicio de expertos (MartínezArias, 2005), que pretende obtener el aporte de valoraciones externas para ofrecer mayor calidad a los elementos que componen el instrumento y una visión imparcial, aparte de la proporcionada por los propios autores en su diseño. En este sentido, se seleccionó un grupo de profesionales en investigación educativa y otros con experiencia en tutorías en el ámbito de la Educación Secundaria. La configuración del grupo de jueces quedó finalmente compuesta por ocho profesionales. Cada juez recibió una plantilla donde se recogía la información del cuestionario inicial de nuestro estudio y sus respectivas dimensiones para ser valorado en una escala de 1 a 5 sobre la relevancia (nivel de significatividad o importancia del ítem respecto a la dimensión en la que se encuadra) y claridad (grado en que el ítem se entiende con facilidad) de los elementos que componen el cuestionario. Por último, el instrumento de validación constaba de un apartado de preguntas abiertas, con la finalidad de proporcionar una información más detallada en relación con la falta de claridad y relevancia de añadir, eliminar o modificar alguno de los ítems presentados.

Una vez recogidas las respuestas del juicio de expertos se siguió una metodología cuantitativa para seleccionar los ítems en función de los criterios de relevancia y claridad, estableciéndose el umbral de rechazo en una puntuación media igual o menor que 4, y aquellos que tuvieran una desviación típica mayor de 1.5 (Cortada de Kohan, 1999). Los resultados obtenidos mostraron ítems con medias (entre 3.50 y 3.88) y desviaciones típicas (entre 1.581 y 2.138) distintas a lo recomendado, por lo que fueron motivo de eliminación, presentado los ítems finales medias que oscilan entre $4.13 \mathrm{y}$ 4.88 y desviaciones típicas entre 0.354 y 1.458 para ambos criterios. Por otra parte, se utilizó una metodología cualitativa para analizar el contenido de aquellas respuestas abiertas de cada ítem o de manera general por cada una de las dimensiones del ins- 
trumento. Según esto, los cambios y correcciones de los ítems fueron principalmente aspectos formales del cuestionario (leves correcciones ortográficas y gramaticales), así como expresiones más adecuadas a la muestra del estudio para mejorar la claridad y la representatividad del indicador al que pertenecen. En la tabla 1 se muestra la configuración final de los ítems incluidos en la escala.

Tabla 1

Dimensiones, ítems y estadísticos descriptivos

\begin{tabular}{|c|c|c|c|}
\hline DIM & ÍTEMS & $\mathbf{M}$ & DT \\
\hline \multirow{13}{*}{ 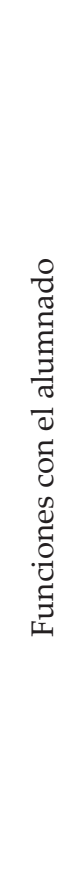 } & $\begin{array}{l}\text { 1. El tutor está preparado para resolver las dudas que le } \\
\text { planteamos los alumnos. }\end{array}$ & 4.049 & 1.021 \\
\hline & 2. El tutor habla con nosotros aunque no lo pidamos. & 3.618 & 1.193 \\
\hline & $\begin{array}{l}\text { 3. El tutor nos sugiere actividades de refuerzo si nos hacen } \\
\text { falta. }\end{array}$ & 3.503 & 1.256 \\
\hline & 4. El tutor se preocupa por nuestros problemas personales. & 3.518 & 1.259 \\
\hline & 5. El tutor habla con la clase cuando surgen problemas. & 4.273 & 0.988 \\
\hline & 6. El tutor contribuye a solucionar los problemas de la clase & 4.019 & 1.093 \\
\hline & $\begin{array}{l}\text { 7. Los alumnos planteamos temas para trabajar en clase du- } \\
\text { rante las tutorías. }\end{array}$ & 2.740 & 1.251 \\
\hline & 8. El tutor sabe tus aficiones, gustos y temas de interés. & 2.584 & 1.105 \\
\hline & 9. Elaboramos las normas de clase con el grupo y el tutor. & 2.784 & 1.386 \\
\hline & 10. El tutor nos ayuda a relacionarnos con los compañeros. & 2.997 & 1.241 \\
\hline & $\begin{array}{l}\text { 11. En las tutorías nos ayudan a sentirnos bien trabajando en } \\
\text { equipo. }\end{array}$ & 3.051 & 1.259 \\
\hline & $\begin{array}{l}\text { 12. En las tutorías me enseñan técnicas de estudio que me } \\
\text { ayuden a aprender mejor. }\end{array}$ & 2.949 & 1.341 \\
\hline & 13. El tutor me motiva para aprender. & 3.354 & 1.244 \\
\hline \multirow{5}{*}{ 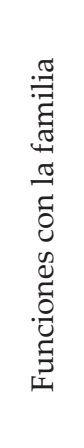 } & $\begin{array}{l}\text { 14. Mis padres participan en actividades que se organizan en } \\
\text { el colegio. }\end{array}$ & 2.717 & 1.004 \\
\hline & $\begin{array}{l}\text { 15. Mis padres colaboran con el tutor en la realización de ac- } \\
\text { tividades. }\end{array}$ & 2.652 & 1.017 \\
\hline & $\begin{array}{l}\text { 16. Mis padres conocen las actividades diarias del colegio a } \\
\text { través de los recursos tecnológicos (teléfono, ordenador, } \\
\text { etc.). }\end{array}$ & 3.105 & 1.472 \\
\hline & 17. El tutor habla con mis padres cuando tengo problemas. & 3.495 & 1.342 \\
\hline & $\begin{array}{l}\text { 18. Los tutores informan a mis padres sobre lo que hago en } \\
\text { clase. }\end{array}$ & 3.418 & 1.333 \\
\hline
\end{tabular}




\begin{tabular}{|c|c|c|c|}
\hline DIM & ÍTEMS & $\mathbf{M}$ & DT \\
\hline \multirow{9}{*}{$\begin{array}{l}\stackrel{0}{\overline{0}} \\
\stackrel{0}{0} \\
0 \\
0 \\
0\end{array}$} & $\begin{array}{l}\text { 19. En las tutorías se llevan a cabo debates sobre temas de ac- } \\
\text { tualidad que me ayudan a pensar sobre los valores de la } \\
\text { vida. }\end{array}$ & 3.044 & 1.341 \\
\hline & $\begin{array}{l}\text { 20. El tutor nos informa sobre temas de nuestro interés (ocio, } \\
\text { salud, etc.). }\end{array}$ & 2.727 & 1.341 \\
\hline & $\begin{array}{l}\text { 21. El tutor nos solicita actividades que requieren el uso de } \\
\text { páginas de internet. }\end{array}$ & 2.605 & 1.287 \\
\hline & $\begin{array}{l}\text { 22. En las tutorías participan profesores del centro para tratar } \\
\text { temas de su especialidad. }\end{array}$ & 2.831 & 1.115 \\
\hline & $\begin{array}{l}\text { 23. En las tutorías participan profesionales de otros centros } \\
\text { y asociaciones para tratar otros temas (educación para la } \\
\text { salud, educación vial, etc.). }\end{array}$ & 2.599 & 1.332 \\
\hline & 24. Utilizo el correo electrónico para preguntar dudas al tutor. & 2.633 & 0.997 \\
\hline & 25. El tutor me ayuda a saber lo que es bueno para mí. & 3.365 & 1.214 \\
\hline & $\begin{array}{l}\text { 26. Las tutorías me ayudan a decidir sobre mi futuro académi- } \\
\text { co (elección de asignaturas, elección de estudios futuros, } \\
\text { etc.). }\end{array}$ & 2.836 & 1.331 \\
\hline & $\begin{array}{l}\text { 27. Las tutorías me ayudan a decidir sobre mi futuro profesio- } \\
\text { nal (conocimiento y elección de profesión, becas, etc.). }\end{array}$ & 2.660 & 1.296 \\
\hline \multirow{10}{*}{ 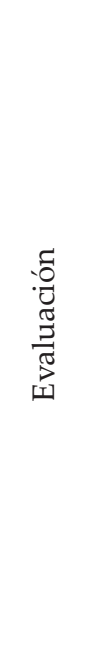 } & $\begin{array}{l}\text { 28. El tutor realiza tutorías en espacios distintos al centro edu- } \\
\text { cativo (parques, mercados, barrio....). }\end{array}$ & 2.628 & 1.080 \\
\hline & $\begin{array}{l}\text { 29. En las tutorías utilizamos dinámicas distintas a otras asig- } \\
\text { naturas (juegos, videos temáticos, etc.). }\end{array}$ & 2.840 & 1.357 \\
\hline & $\begin{array}{l}\text { 30. Estoy interesado por aquellos temas que trabajamos en las } \\
\text { tutorías }\end{array}$ & 3.194 & 1.150 \\
\hline & $\begin{array}{l}\text { 31. Me siento cómodo con las actividades que realizamos en } \\
\text { las tutorías. }\end{array}$ & 3.533 & 1.176 \\
\hline & 32. Opinamos sobre las actividades realizadas en las tutorías. & 2.981 & 1.277 \\
\hline & 33. Estoy satisfecho con el funcionamiento de las tutorías. & 3.390 & 1.252 \\
\hline & 34. Pienso en el trabajo que realizo en las tutorías. & 2.681 & 1.279 \\
\hline & 35. Valoro las tutorías que se realizan en clase & 3.214 & 1.239 \\
\hline & 36. Las tutorías me ayudan a mejorar como persona. & 3.004 & 1.321 \\
\hline & 37. Pongo en práctica lo que aprendo en las tutorías. & 2.794 & 1.274 \\
\hline
\end{tabular}

\section{Procedimiento}

El cuestionario fue aplicado durante el curso escolar 2016/2017 (enero - abril). Se contactó con los centros de la CAM mediante el envío de un correo electrónico donde se explicaba brevemente la investigación y se solicitaba su colaboración. Para aquellos centros que manifestaban su interés en participar en el estudio se concertó una cita personal con el director del centro, en la que se presentaba con más detalle 
el instrumento del estudio. Se entregaba sobres con 30 cuestionarios en función de los grupos de clase que contaba el centro. Para el seguimiento de los cuestionarios se facilitó una fecha orientativa y se mantuvo contacto a través del teléfono y el correo electrónico con la persona asignada en cada centro para la recogida final de la información (aproximadamente dos semanas). De igual modo, se garantizaba el anonimato y confidencialidad de los datos.

\section{Análisis de datos}

Se estudió la fiabilidad del instrumento mediante el cálculo del alfa de Cronbach con el programa informático estadístico SPSS versión 21, así como el análisis de la capacidad de discriminación de los ítems a través del índice de homogeneidad (correlación elemento total-corregida). Este indicador lo que refleja es el grado que un ítem mide lo mismo que la prueba global, es decir, el grado de conexión con el instrumento. Los valores inferiores a 0.2 determinan que se suprima el ítem (Hair et al., 2014).

De igual modo, para la validación del constructo se determinó el ajuste de bondad del modelo inicial propuesto a nivel teórico a través de la herramienta informática IBM SPSS AMOS versión 21 que corresponde a las dimensiones anteriormente expuestas. Se utilizó el Análisis Factorial Confirmatorio (AFC) para cuya aplicación e interpretación nos basaremos en las indicaciones de Byrne (2010), Kline (2010) y Hair et al. (2014): CMIN/DF entre 2 y 5, CFI e IFI > 0.9, PRATIO, PNFI y PCFI > 0.7, RMSEA <0.06, y HOELTER>200).

\section{Resultados}

\section{Fiabilidad y análisis de los ítems}

El estudio de la fiabilidad se ha obtenido a través de la consistencia interna del instrumento, calculando el Coeficiente de Alfa $(\alpha)$ de Cronbach, cuyo resultado inicial fue excelente, 0.927. Al analizar los ítems se tomó la decisión de eliminar el ítem 28 por presentar un nivel inferior a 0.2 en el índice de homogeneidad (Hair et al., 2014), obteniendo un $\alpha$ de 0.928 . Igualmente, se estudió la fiabilidad, de acuerdo a los resultados del AFC, obteniendo un valor final excelente y valores aceptables por dimensiones ( $\alpha$ global: 0.929, $\alpha$ dimensión Funciones con el alumnado: 0.868, $\alpha$ dimensión Funciones con las familias: 0.649, $\alpha$ dimensión Desarrollo: 0.797, $\alpha$ dimensión Evaluación: 0.876).

Asimismo, se estudió la variabilidad y tendencia central de los ítems con la intención de encontrar algún comportamiento irregular. Para ello, se realizó un estudio inicial descriptivo de las respuestas con medias que oscilan entre 2.584 y 4.273, y desviaciones típicas entre 0.988 y 1.472. Así pues, los resultados obtenidos fueron adecuados (tabla 1).

\section{Validez de constructo (Análisis Factorial Confirmatorio)}

El AFC se llevó a cabo mediante la técnica de modelos de ecuaciones estructurales en lo que se conoce como modelo de medida a través de la metodología S.E.M. (Structural Equation Modeling). El objetivo del empleo de esta técnica es analizar las relaciones 
existentes entre las variables y los factores correspondientes a nuestra estructura o modelo de medida formulado inicialmente y elaborado en base a la fundamentación teórica consultada.

Comenzamos mostrando los resultados obtenidos en las fases del proceso de modelización estructural a través de la definición del modelo teórico y la especificación de las reglas de correspondencia entre las variables (latentes y observables) de nuestro instrumento. Se utiliza el análisis de estructuras de covarianza para demostrar si los ítems incluidos en el instrumento explican el factor al que pertenecen. Por ello, se propuso el modelo inicial de medida (Figura 1), que consta de 4 variables latentes (FA: Funciones con el alumnado, FF: Funciones con la familia, DE: Desarrollo y EVA: Evaluación), 36 variables observadas (FA01 a EV37, respetando la numeración de las variables de la tabla 1) y 36 términos de error (de e01 a e37). Del mismo modo, se definen 36 pesos de regresión entre los ítems y su factor, que se suelen denominar cargas factoriales, además se incluyen los 36 pesos de regresión entre los términos de error y sus variables asociadas, que indican el efecto del error de medida sobre la variable. Por último, se incluyeron cuatro correlaciones entre los factores latentes correspondientes a las dimensiones de la escala.

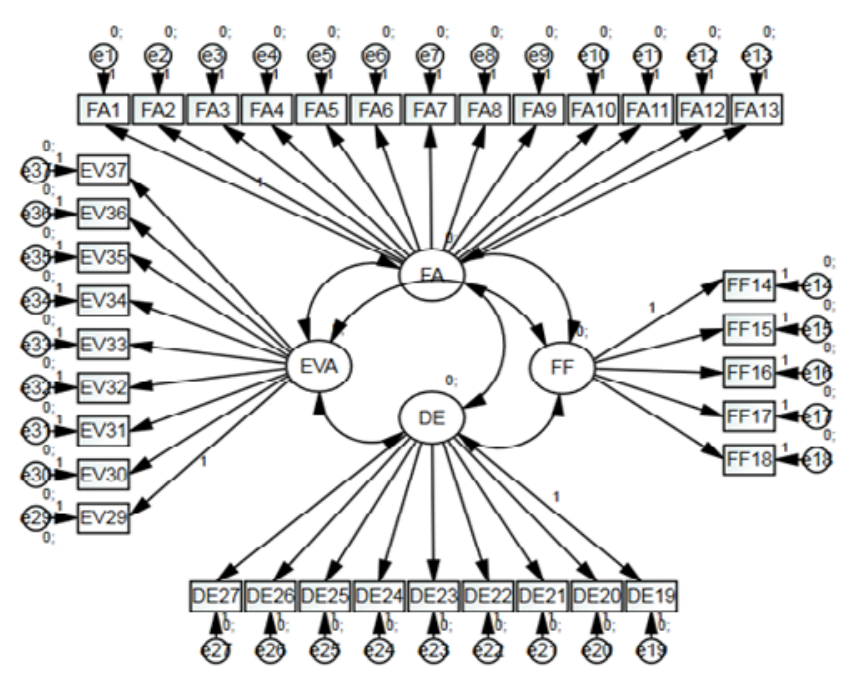

Figura 1. Modelo estructural inicial de medida sobre las tutorías en los centros educativos

Al especificar el modelo, y comprobada la normalidad multivariada (Coeficiente de Mardia=181.746 menor que $\mathrm{p} \cdot(\mathrm{p}+2)$, siendo $\mathrm{p}$ el número de variables observadas, $36 \cdot(36+2)=1368)$ (Bollen, 1989), se procedió a la estimación de parámetros por el procedimiento de Máxima Verosimilitud (ML) por ser el más eficiente y no sesgado cuando se cumplen los supuestos de normalidad multivariante, y lo suficientemente robusto como para no verse afectado por ligeras oscilaciones en función de la distribución normal multivariada (Lévy \& Valera, 2003). Los resultados obtenidos en los índices de ajuste del modelo son los siguientes: $\mathrm{CFI}=0.831$ y de $\mathrm{IFI}=0.830$ por debajo del valor requerido 0.90, según Kline (2010), debido, en parte, a cargas factoriales insatisfactorias 
de los ítems 7, 14, 15, 21, 22, 23, 24 (valores inferiores a 0.5 señalado por Byrne, 2010), por lo que dichos ítems fueron eliminados.

Se analizaron los índices de modificación, donde se comprobó la posibilidad de incluir algunas covariaciones entre términos de error que reducen notablemente el estadístico chi-cuadrado, siendo justificadas desde el punto de vista teórico. Así pues, se decidió incluir dos variables latentes: los factores Resolución de Problemas (RP, comprende la formación y mediación de los problemas) y Competencias desde el Aula (CA, ligadas al trabajo en equipo, desarrollo de habilidades sociales, autorregulación de su aprendizaje, el respeto y convivencia en el aula) incluidos en la dimensión Funciones con el alumnado.

Por otro lado, los índices de modificación desvelaron la conveniencia de algunas correlaciones entre términos de error que son factibles desde un punto de vista teórico, y que reducirían ostensiblemente el estadístico chi-cuadrado, tales como: entre e2 y e4 (ambos indicadores hacen referencia a la atención por parte del tutor hacia los estudiantes), entre e12 y e13 (sus elementos aluden a un mejor aprendizaje del estudiante), entre e19 y e20 (sus elementos indican actividades que tratan temas actuales y de interés), entre e26 y e27 (sus indicadores se centran en la toma de decisiones académicas y profesionales), entre e31 y e33 (valoran aspectos personales en relación a la satisfacción y comodidad de las tutorías) y entre e36 y e37 (hacen referencia a los resultados de mejora y transferencia de las actividades realizadas en las tutorías) (Tabla 2).

Tabla 2

Índices de modificación

\begin{tabular}{ccccc}
\hline & & & M.I. & Par Change \\
\hline e26 & $<->$ & e27 & 1468.576 & 0.588 \\
e36 & $<->$ & e37 & 330.909 & 0.243 \\
e31 & $<->$ & e33 & 194.323 & 0.181 \\
e19 & $<-->$ & e20 & 184.607 & 0.248 \\
e12 & $<->$ & e13 & 74.023 & 0.144 \\
e2 & $<->$ & e4 & 67.499 & 0.127 \\
\hline
\end{tabular}

Tras las modificaciones señaladas, se obtiene un modelo recursivo (Figura 2), estimado sobre una muestra de 4254 sujetos, con 66 variables: 29 variables observadas (correspondientes a los ítems) y 37 variables latentes (6 factores, 29 términos de error y 2 términos de perturbación). De esas 66 variables, 33 son exógenas (29 términos de error, 2 términos de perturbación y 2 factores), y 33 son endógenas (29 indicadores y 4 factores). Por otro lado, son 101 parámetros a ser estimados, por lo que el modelo consta de 363 grados de libertad, resultando un modelo sobreidentificado y con posibilidad de ser estimado.

En las tablas correspondientes a la estimación de parámetros, se puede comprobar la elevada significación estadística de todos ellos. Con respecto a los índices de 
modificación no se encuentra ninguno que sea relevante para tenerlo en cuenta. Los índices de ajuste son la mayoría bastante satisfactorios, destacando un CFI=0.940 y un $\mathrm{IFI}=0.940$. Respecto a los residuos, se alcanza un RMSEA de 0.044 y el tamaño muestral es adecuado, pues el índice de Hoelter es de 544 (>200). Asimismo, los índices de parsimonia son elevados (PRATIO=0.894, PNFI=0.834, PCFI=0.840), por lo que podemos afirmar que nos encontramos con un modelo de medida bastante parco. En cambio, el chi-cuadrado (9.244) no cumple con el criterio de aceptación establecido por Kline (2010), sin embargo, siguiendo las indicaciones de Ruiz, Pardo y Sanmartín (2010), justifican dicho resultado debido a la sensibilidad que presenta al tamaño de la muestra, por lo que relacionan el estadístico chi-cuadrado con los grados de libertad del modelo, y si su valor es inferior a 3, el ajuste es adecuado ( $\chi 2$ / $\mathrm{gl}=9.244 / 363=0.0254$ ) (Tabla 3).

Tabla 3

Índices de ajuste: modelo inicial y modelo final de medida

\begin{tabular}{ccccc}
\hline Medida & $\begin{array}{c}\text { Nivel de ajuste } \\
\text { recomendado }\end{array}$ & Modelo inicial & Modelo final & Aceptabilidad \\
\hline $\begin{array}{c}\text { CMIN/DF } \\
\chi 2 / \text { gl }\end{array}$ & $\begin{array}{c}2-5 \\
<3\end{array}$ & 16.151 & $\begin{array}{c}9.244 \\
*(0.0254)\end{array}$ & Aceptable* \\
\hline IFI & $>0.9$ & 0.831 & 0.940 & Buena \\
CFI & $>0.7$ & 0.830 & 0.940 & Muy buena \\
PRATIO & & 0.935 & 0.894 & Muy buena \\
PNFI & 0.768 & 0.834 & Muy buena \\
PCFI & $<0.06$ & 0.777 & 0.840 & \\
\hline RMSEA & $>200$ & 0.60 & 0.044 & 518 \\
\hline HOELTER.05 & & 289 & 544 & \\
HOELTER.01 & & 300 & & \\
\hline
\end{tabular}

En el modelo expuesto (Figura 2) podemos analizar los valores estandarizados de los parámetros donde se constata la buena calidad de los indicadores, ya que las cargas factoriales son superiores a 0.5 , exceptuando dos cargas correspondientes a los indicadores EV29 y FF16 inferiores a 0.5, pero que mantenemos en el modelo por su relación con la fundamentación teórica. La correlación entre los términos de error toma un valor estimable en todos los casos (la menor es 0.14). Por último, observamos que los cuatro componentes muestran correlaciones altas coherentes con la práctica de las tutorías en las aulas. Asimismo, se observa un nivel de correlación moderado entre el componente Funciones con la Familia (FF) y el resto, ya que dichas funciones no suelen ser percibidas por el alumnado durante el desarrollo de las tutorías, salvo que se trabajen de manera específica. 


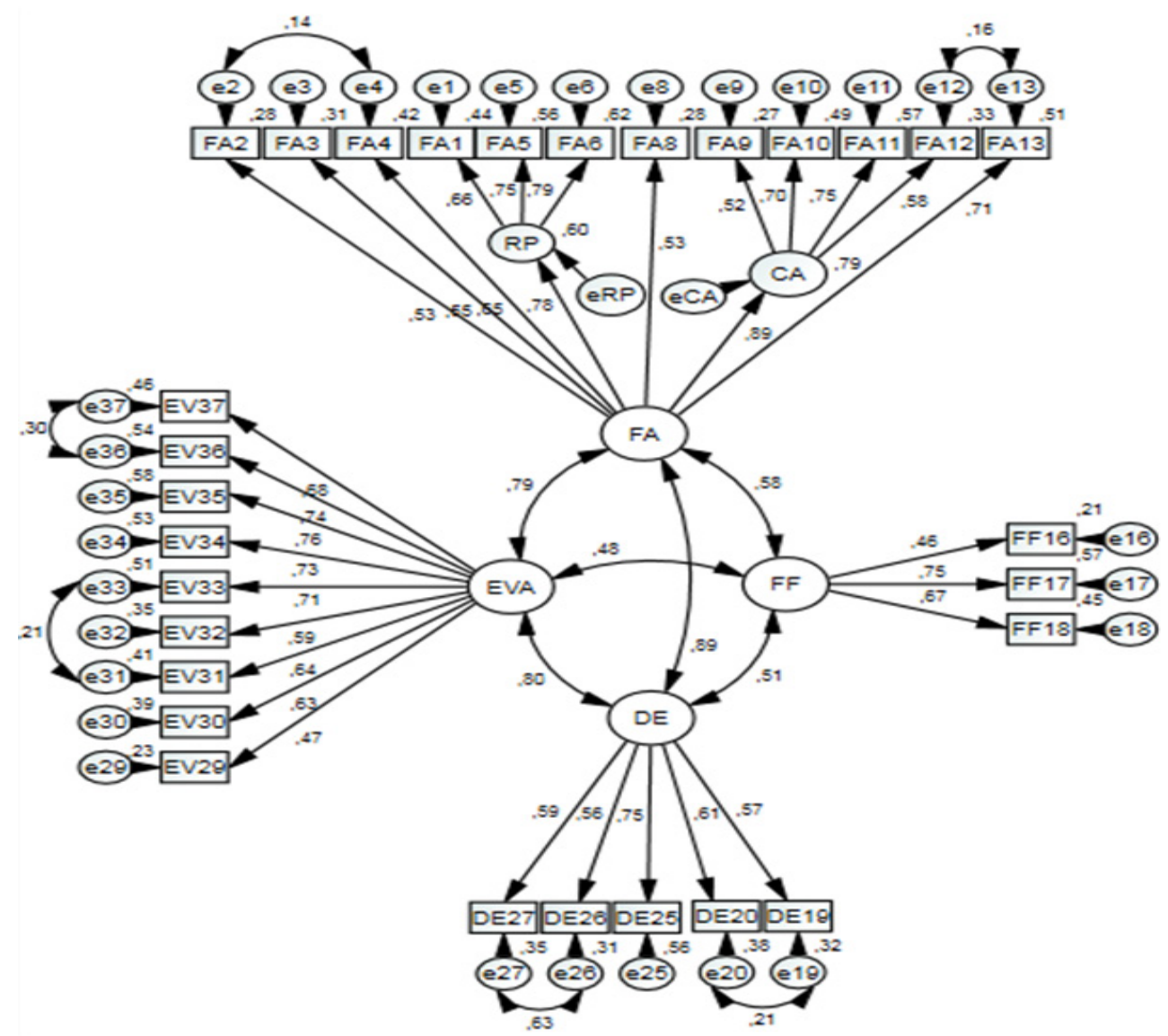

Figura 2. Modelo estructural final de medida sobre las tutorías en los centros educativos

\section{Conclusiones}

Los resultados han permitido mostrar el diseño de un instrumento de medida sobre la valoración de las tutorías destinado a estudiantes de Educación Secundaria Obligatoria y Posobligatoria, fundamentado teóricamente y definido operativamente, teniendo en cuenta cuatro dimensiones: Funciones con el Alumnado, Funciones con las Familias, Desarrollo y Evaluación de las tutorías. Se trata de una definición de la práctica tutorial relevante y actual apoyada por un planteamiento teórico como en la operativización de los ítems, ambos consistentes al conjunto del instrumento. Asimismo, se constituye como una herramienta de bastante utilidad para los centros educativos y sus profesionales con la posibilidad de atender las demandas y necesidades de los estudiantes.

El tratamiento riguroso de la fundamentación teórica, ha permitido obtener una fiabilidad global del instrumento excelente y una consistencia interna adecuada. La dimensión Funciones del Tutor con la Familia presenta un valor de fiabilidad más 
modesto, siendo una posible explicación la falta de trabajo conjunto entre estudiantes - familia - tutor durante el desarrollo de las tutorías. Este hecho, desde el punto de vista teórico se tiene presente en la práctica tutorial, como señalan López y Pantoja (2016), con la importancia de crear un vínculo más estrecho familia - escuela desde el conocimiento y tareas de las tutorías con la intención de ir participando asiduamente en la formación de sus hijos. No obstante, cabe señalar que las dimensiones definidas y propuestas se sustentan en la literatura al respecto, tanto en planteamientos teóricos como en las investigaciones, por lo que se justifica una buena validez de contenido, avalada por expertos.

Por otro lado, la validez de constructo, explicado a través del AFC, muestra una calidad métrica adecuada y un ajuste satisfactorio del modelo. Asimismo, la adecuación de los ítems que componen la escala establece la relación con la teoría estudiada y los ítems que derivaron de ella. En este sentido, el AFC confirma la configuración multidimensional del instrumento y el valor de poder medir diferentes aspectos correlacionados entre sí, teniendo en cuenta que cada uno mide características diferentes de las tutorías. No obstante, el tipo de muestreo de carácter incidental, procedimiento común en este tipo de estudios, puede limitar el poder de generalización de los resultados en relación con la adecuación de las variables sociodemográficas de la población.

La validación del AFC ha permitido probar que la técnica es plausible y mostrar la sólida configuración multidimensional del instrumento y el valor de medir diferentes dimensiones correlacionadas entre sí que subyacen del estudio: las Funciones del Tutor con el Alumnado (compuesta por Resolución de Problemas y Competencias desde el Aula), Funciones del Tutor con las Familias, Desarrollo de las Tutorías y Evaluación de las Tutorías. Este trabajo contribuye al análisis y estudio de ciertas cuestiones de diverso tipo relacionadas con la acción tutorial (a nivel escolar, personal o profesional, como elementos fundamentales según Alegre et al., 2017). Aun así, todavía hay mucho trabajo por hacer, y seguir profundizando en las diferentes tareas tutoriales que se llevan a cabo en la formación formativa de los estudiantes. También es necesario centrarnos en otras líneas de trabajo que permitan definir la capacitación del profesorado en el manejo de grupos de clases (Hudson et al., 2009) y la elección del tutor acorde a criterios adecuados como parte de la responsabilidad del profesorado para ejercer la función tutorial con cualquier estudiante, para ello, el tutor debe poseer conocimientos sobre su profesión y una madurez personal y emocional adecuada en beneficio del desarrollo integral del alumnado (González-Benito \& Vélaz de Medrano, 2014; Martínez Clares et al., 2016). De igual modo, hay que tener presente la limitación de la dimensión funciones del tutor con la familia profundizando en el trabajo conjunto de la familia-(alumnado)-escuela proporcionando nuevos indicadores que favorezca el trabajo de las tutorías con los estudiantes.

En síntesis, el instrumento propuesto contribuye al estudio de las tutorías en los centros educativos atendiendo a las funciones y actividades del proceso formativo de los propios estudiantes, siendo bastante relevante debido a la escasez de instrumentos centrados en dichos aspectos (De la Cruz \& Abreu-Hernández, 2017), tanto desde un punto de vista teórico (la robustez y solidez proporcionada a través de la elaboración basada en dimensiones), como por sus características psicométricas relativas a su 
fiabilidad y validez. Igualmente, el estudio llevado a cabo se diferencia del resto por tratar las tutorías en la educación básica en un tiempo donde las investigaciones en la formación universitaria abarcan la mayor parte de la literatura científica en el ámbito de la acción tutorial. Esto, unido a la consistencia del instrumento obtenida a través de la validez de constructo y la estructura dimensional propuesta, y acorde al objetivo de nuestro estudio, se puede afirmar que se ha contribuido con ello aportando conocimiento a la comunidad científica y profesional sobre un elemento clave de la orientación educativa como son las tutorías.

\section{Referencias}

Aguaded, E. (2014). Procesos de planificación y desarrollo de la Acción Tutorial. En J. Expósito (Coord.), La acción tutorial en la educación actual (pp.95-116). Madrid: Síntesis. Alegre, O.M., Guzmán, R., \& Arvelo, C.N. (2017). La tutoría y la inclusión en la formación del profesorado de educación primaria. Educatio Siglo XXI, 35(2), 43-64. doi: https://doi.org/10.6018/j/298511

Álvarez González, M., \& Bisquerra, R. (2012). Orientación educativa. Modelos, áreas estrategias y recursos. Madrid: Wolters Kluwer.

Álvarez Justel, J. (2017). La tutoría en Secundaria. Educatio siglo XXI, 35(2), 65-90. doi: https://doi.org/10.6018/j/298521

Anaya, D., Pérez-González, J.C., \& Suárez, J.M. (2011). Los contenidos de formación de los profesionales de la orientación educativa desde la perspectiva de los propios orientadores. Revista de Educación, (356), 607-629. doi: 10-4438/1988-592X-RE-2010356-053

Arza, N., De Salvador, X., \& Mascarenhas, S. (2014). La función tutorial del profesorado: estudio evaluativo en tres universidades federales brasileñas. Revista Electrónica Interuniversitaria de Formación Del Profesorado, 17(3), 105-121. doi: http://dx.doi. org/10.6018/reifop.17.3. 204081

Bollen, K.A. (1989). Structural equations with latent variables. New York: Wiley

Byrne, B.M. (2010). Structural equation modeling with AMOS: Basic concepts, applications, and programming. Mahwah, NJ: Erlbaum.

Caldera, J.F., Carranza, M.R., Jiménez, A.A., \& Pérez, I. (2015). Actitudes de los estudiantes universitarios ante la tutoría. Diseño de una escala de medición. Revista de la Educación Superior, 44(173), 103-124. doi: https://doi.org/10.1016/j.resu.2015.04.004

Castellano, E.A., \& Pantoja, A. (2015). Las Tecnologías de la Información y la Comunicación (TIC) como recurso en la Acción Tutorial de Primaria. Revista Científica Electrónica de Educación y Comunicación en la Sociedad Del Conocimiento, 2(15), 350-378. Recuperado de https://dialnet.unirioja.es/servlet/articulo? codigo=5476876

Cortada de Kohan, N. (1999). Teorías psicométricas y Construcción de Tests. Buenos Aires: Editorial Lugar.

De la Cruz, F., \& Abreu-Hernández, L.F. (2017). Evaluación de la tutoría en los estudios de posgrado: construcción y validez de escalas. Revista de Docencia Universitaria, 15(1), 11-36. doi: https://doi.org/10.4995/redu.2017.5682

Díaz de Greñu, S., \& Parejo, J.L. (2013). La promoción de la igualdad y el respeto de la diversidad afectivo-sexual: bases de un programa de orientación y tutoría para 
educación secundaria. Revista Española de Orientación Y Psicopedagogía, 24(3), 63-79. Recuperado de https://www.redalyc.org/articulo.oa?id=338230795005

García Nieto, N. (2011). La función tutorial en el ámbito educativo. Padres y Maestros, 342, 5-9. Recuperado de https://revistas.comillas.edu/index.php/padresymaestros / article/view/310/239

Gil-Albarova, A., Martínez, A., Tunnicliffe, A., \& Moneo, J.M. (2013). Estudiantes universitarios y calidad del plan de acción tutorial. Valoraciones y mejoras. Revista de Docencia Universitaria, 11(2), 163-176. doi: https://doi.org/10.4995 /redu.2013.5567

González-Benito, A., \& Vélaz de Medrano, C. (2014). La acción tutorial en el sistema escolar. Madrid: UNED.

Hair, J.F, Black, W.C., Babin, B.J., \& Anderson, R.E. (2014). Multivariate data analysis (Seventh edition, Pearson new international edition). Harlow, Essex: Pearson Education Limited.

Hudson, P., Usak, M., \& Savran-Gencer, A. (2009). Employing the five-factor mentoring instrument: Analysing mentoring practices for teaching primary science. European Journal of Teacher Education, 32(1), 63-74.

Jiménez, M.A., \& Navaridas, F. (2012). Cómo son y qué hacen los "maestros excelentes": la opinión de los estudiantes. Revista Complutense de Educación, 23(2), 463-485. Recuperado de https://dialnet.unirioja.es/servlet/articulo?codigo $=4134106$

Kline, R.B. (2010). Principles and practice of structural equation modeling. New York: Guilford Press.

Lévy, J.P., \& Varela, J. (2003). Análisis multivariable para las ciencias sociales. Madrid: Pearson Educación.

López, M., \& Pantoja, A. (2016). Diseño y validación de una escala para comprobar la percepción y satisfacción de las familias andaluzas en relación con los procesos tutoriales en centros de Educación Primaria. Revista Española de Orientación Y Psicopedagogía, 27(1), 47-66. Recuperado de http://revistas.uned.es/index.php /reop/ article/view/17007/14594

Lozano, J.R. (2012). Hándbol, la enseñanza del deporte a través del método global. Barcelona: Paidotribo.

Martínez-Arias, R. (2005). Psicometría: teoría de los test psicológicos y educativos. Madrid: Editorial Síntesis.

Martínez Clares, P., Martínez Juárez, M., Pérez Cusó, F.J., González Morga, N., \& González Lorente C. (2016). Manual de orientación y acción tutorial. Murcia: Diego Marín Librero Editor.

Monge, C. (2010). Tutoría y orientación educativa. Nuevas competencias. Madrid: Wolters Kluwer.

Pérez Cusó, F.J., Martínez Clares, P., \& Martínez Juárez, M. (2015). Satisfacción del estudiante universitario con la tutoría. Diseño y validación de un instrumento de medida. Estudios sobre educación, 29, 81-101. Recuperado de http://dadun.unav.edu/ handle/10171/39803

Rodríguez Fernández, S. \& Romero, M. (2015). La función tutorial en Educación Infantil y Primaria: desempeño profesional del profesorado. Revista Electrónica Interuniversitaria de Formación del Profesorado, 18(2), 43-55. doi: http://dx.doi.org/10.6018/ reifop.18.2.219131 
Rosiles, L., Macías, E., Pérez, S., \& León, J.C. (2013). La competitividad del programa de tutorías académicas desde la percepción de los alumnos. Revista Global de Negocios, 8(2), 105-116.

Rubio, P.P., \& Martínez, J.F. (2012). La acción tutorial desde la perspectiva de los alumnos de la Universidad Autónoma de San Luis Potosí. Perfiles Educativos, 34(138), 28-45. Recuperado de http://scielo.unam.mx/pdf/peredu/v34n138 /v34n138a3.pdf

Ruiz, M.A., Pardo, A., \& Sanmartín, R. (2010). Modelos de ecuaciones estructurales. Papeles del Psicólogo, 31(1), 34-45. Recuperado de http://www.redalyc.org/pdf /778/77812441004.pdf

Sáiz-Manzanares, M.C., Bol-Arreba, A., \& Payo-Hernanz, R.J. (2014). Validación de una Escala de Evaluación de Tareas de Tutoría en la Universidad. Electronic Journal of Research in Educational Psychology, 12(3), 835-852. doi: http://dx.doi.org/10.25115/ ejrep.34.14027

Sánchez García, M.F. (2013). Orientación profesional y personal. Madrid: UNED.

Sánchez-Porro, D.G., \& González, E. (2017). Los programas de mediación escolar como herramientas para la promoción de unas relaciones de pareja saludables en la adolescencia. Revista Española de Orientación y Psicopedagogía, 28(1), 72-85. doi: http:// doi-org/10.5944/reop.vol.28.num.1.2017.19359

Santana, L.E., García, L.F., \& Cruz, A. (2010). El Programa de Orientación Educativa y Sociolaboral: un instrumento para facilitar la toma de decisiones en Educación Secundaria. Revista de Educación, (351), 73-105. Recuperado de http://www.revistaeducacion.educacion.es/re351/re351_04.pdf

Solaguren-Beascoa, M., \& Moreno, L. (2016). Escala de actitudes de los estudiantes universitarios hacia las tutorías académicas. Revista Española de Orientación y Psicopedagogía, 19(1), 247-266. Recuperado de http://revistas.uned.es/index.php /educacionXX1/article/view/15586

Torrecilla, E.M., Rodríguez, M.J., Herrera, M.E., \& Martín, J.F. (2013). Evaluación de calidad de un proceso de tutoría de titulación universitaria: La perspectiva del estudiante de nuevo ingreso en educación. Revista Española de Orientación y Psicopedagogía, 24(2), 79-99. Recuperado de http://revistas.uned.es/index.php/reop/article/ viewFile/11260/10784

Urosa, B., \& Lázaro, S. (2017). La función tutorial en educación infantil y primaria: actividades que implica y dificultad percibida por el profesorado en su desarrollo. Educatio Siglo XXI, 35(2), 111-138. Recuperado de https://digitum.um.es/digitum/ bitstream/10201/54383/2/298541-1025891-1-PB.pdf

Youde, A. (2016). Tutor emotional competences valued by learners in a blended learning context. European Journal of Open, Distance and e-learning, 19(2), 81-97. doi: https:// doi.org/10.1515/eurodl-2016-0008

Fecha de recepción: 9 de octubre de 2018.

Fecha de revisión: 10 de octubre de 2018.

Fecha de aceptación: 26 de febrero de 2019. 
